\title{
Emerging Data on Fistula Laser Closure (FiLaC) for the Treatment of Perianal Fistulas; Patient Selection and Outcomes
}

\author{
Samuel O Adegbola $\mathbb{D}^{1,2}$ \\ Kapil Sahnan ${ }^{1,2}$ \\ Phillip Tozer ${ }^{1,2}$ \\ Janindra Warusavitarne ${ }^{1,2}$ \\ 'Robin Phillips Fistula Research Unit, St \\ Mark's Hospital and Academic Institute, \\ Harrow, UK; ${ }^{2}$ Department of Surgery \\ and Cancer, Imperial College, \\ London, UK
}

\begin{abstract}
Fistula laser closure (FiLaC) is a relatively new sphincter-sparing technique in fistula surgery that was initially reported in 2011. It involves the radial dissipation of laser energy in the fistula tract and, through a combination of coagulation and shrinkage of the tract, is proposed to result in progressive sealing of fistulas. Early studies have suggested minimal impact on continence and touted the advantage of minimal morbidity with potential of repeat procedures if the technique fails initially. Despite early promising results, ten years on, questions remain on the technique, patient selection and long-term outcomes. This narrative review assesses the evidence reported to-date of radially emitting laser fistula surgery in the treatment of perianal fistulas.
\end{abstract}

Keywords: fistula, surgery, FiLaC, fistula laser closure, perianal fistula, perianal Crohn's disease

\section{Introduction}

The modern surgical approach to anal fistulas includes several sphincter-saving procedures, which offer an attempt at cure whilst minimising morbidity, especially the impact on continence. Coloproctologists now have an armamentarium of options, which includes the closure of the fistula tract with plugs, fibrin glue, or collagen paste without fistulotomy (ie, laying open) or by means of fistulectomy (ie, core-out technique). Further sphincter-saving options include advancement flaps, ${ }^{1}$ LIFT (ligation of the intersphincteric fistula tract), ${ }^{2}$ VAAFT (video-assisted anal fistula treatment $)^{3}$ and laser ablation procedures. ${ }^{4}$ Varying success rates and lack of long-term data mean that there is no one universally agreed gold standard and thus treatments are assessed by a combination of patient and surgeon factors. ${ }^{5}$

In 2011 Wilhelm reported on the use of a novel diode laser source and radial emitting laser probe to obliterate the fistula tract throughout its length from within. ${ }^{4}$ Its conceptual development was based on varicose vein laser treatment and follows the same principle of limiting penetration and uniformly distributing photothermal energy to ensure homogeneity. The thermal energy generated from the laser is dissipated radially, which is different to previous lasers used in coloproctology such as the neodymium yttrium aluminium garnet (YAG) laser ${ }^{6}$ or the $\mathrm{CO}^{2}$ laser $^{7}$ where linear energy was used. The aim is the destruction of the granulation tissue and the epithelial cells through a combination of coagulation and shrinkage of the tract. The thermal energy acts on proteins within the tissue, disrupting the structure and supposedly aids
Correspondence: Samuel O Adegbola St. Mark's Hospital, Watford Road,

Harrow, HAI 3UJ, UK

Tel +442088695328

Email samadeg@doctors.net.uk 
the sealing effect. ${ }^{8}$ It is also thought that better accuracy of the laser (in comparison to electrocautery) decreases the risk of damage to surrounding structures (ie, anal sphincters).

In this paper we review the evidence available on this novel technique and assess its emerging role in fistula surgery.

\section{Methods}

All articles published in the English literature in peerreviewed journals on $\mathrm{FiLaC}^{\mathrm{TM}}$ in patients with anorectal fistula were considered. A systematic search using MEDLINE and Embase databases was performed through to 18 March 2021, using the MeSH terms "fistula", "laser", "surgery" and "fistula tract laser closure" and including relevant sub-classifications. The obtained studies were supplemented with searches of reference lists and bibliographies of selected articles in order to ensure that no relevant articles were missed during the original searches. All relevant studies on FiLaC describing the patient population were included in this review. Any discrepancies were discussed between the independent assessors to reach agreeable consensus. Collected data were expressed in spreadsheet format (using Microsoft Excel, Microsoft, Redmond, WA) and analysed to ascertain any possible conclusions from their collective information. Exclusion criteria were case reports, systematic reviews, commentaries (without new data) and studies published with overlapping data from centres/authors at multiple time points (to avoid duplicity).

Data collected for each study included number of patients, age range/mean, gender distribution, success of operation (ie, healing of fistula), duration of follow-up and endpoints, including complications. These were expressed as totals, percentages and in descriptive terms, as applicable.

\section{Results}

The literature search revealed 14 published studies ${ }^{9-22}$ on FiLaC after excluding some studies; amongst these were two systematic reviews, both published in $2020^{23,24}$ (see Figure 1 for search strategy). Since these reviews, however, there have been more recent studies ${ }^{9,10,12,13,17-20}$ including larger series ( $\mathrm{n}>80),{ }^{9,13,18}$ as well as a study assessing FiLaC in solely Crohn's-related perianal fistulas. ${ }^{20}$ Table 1 illustrates the studies assessed and their characteristics when evaluating FiLaC for treatment of perianal fistulas. Study numbers varied from 10-117, with follow up ranging from 2-87 months. All published data were retrospective case series, albeit with largely prospectively collected data.

\section{Patient Selection}

Predominantly male patients were included in the studies and reported median/mean ages were fairly similar (ie, 3550 year olds), although there was a wide range of those included (17-88 year olds). The majority of studies involved patients with previous attempted fistula surgeries, with only one study reporting on patients $(n=20)$ without prior fistula surgery. ${ }^{17}$ Six studies ${ }^{9,11,12,15,17,18}$ reported a significant majority $(>75 \%)$ of treated patients having a seton drainage prior to $\mathrm{FiLaC}$, although the duration was not always specified. The technique has been demonstrated in simple, complex, and recurrent fistula. Fistula

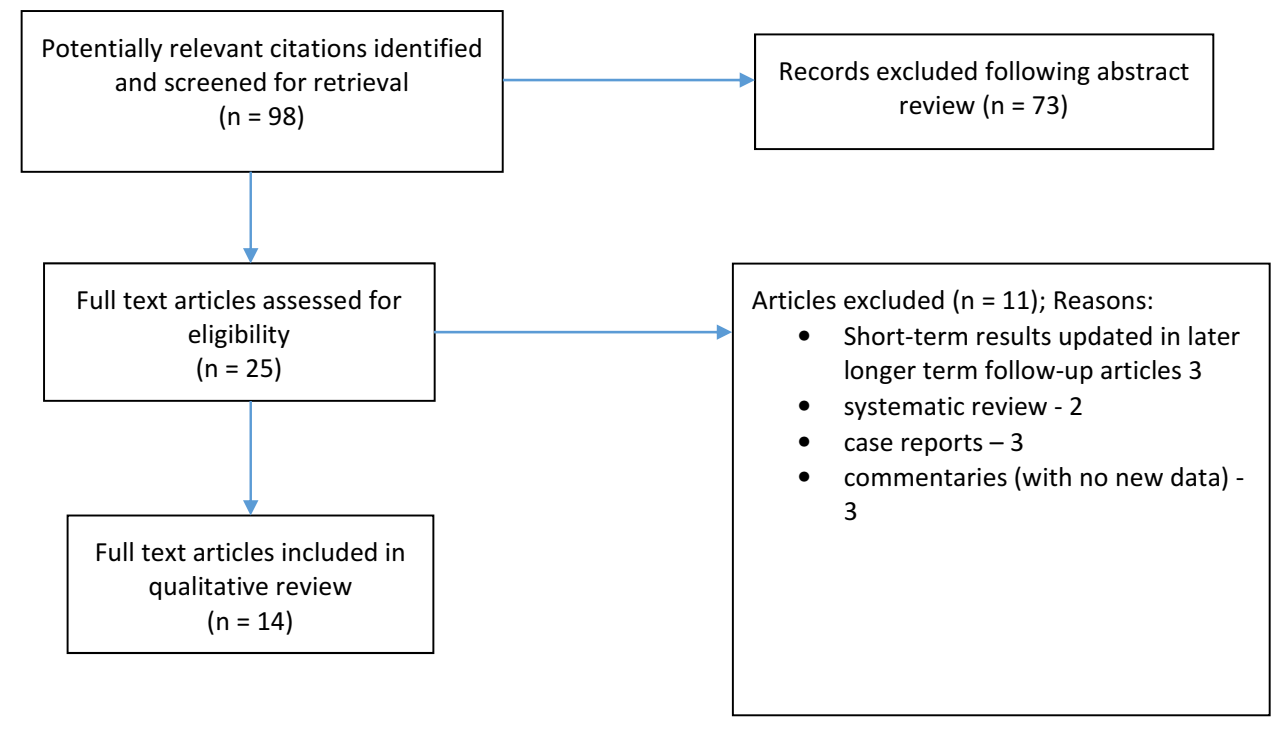

Figure I Flow diagram of search strategy. 
Table I Demographics for FiLaC in the 14 Retrospective Studies

\begin{tabular}{|c|c|c|c|c|c|c|}
\hline Study & Country & Number & $\begin{array}{l}\text { Number of } \\
\text { Males (\%) }\end{array}$ & $\begin{array}{c}\text { Age in Years } \\
\text { Median (Range) }\end{array}$ & $\begin{array}{c}\text { Crohn's } \\
\text { Disease } \\
(\%)\end{array}$ & $\begin{array}{c}\text { Median Duration of } \\
\text { Follow-Up in Mths (Range) }\end{array}$ \\
\hline $\begin{array}{l}\text { Nordholm-Carstensen et al } \\
(2021)^{12}\end{array}$ & Denmark & 66 & $28(42)$ & $40^{*}$ & II (16) & $19(12-26)$ \\
\hline Isik et al $(2020)^{13}$ & Turkey & 100 & $72(72)$ & $42(21-83)$ & $0(0)$ & $48(6-56)$ \\
\hline Wolicki et al $(2020)^{18}$ & Germany & 83 & $64(77.1)$ & $50.01(14.59)^{*}$ & $2(2.4)$ & $41.99(4-87)^{*}$ \\
\hline de Bonnechose et al $(2020)^{9}$ & France & 100 & $65(65)$ & $43(22-88)$ & $10(10)$ & $13.6(6-23)$ \\
\hline Serin et al $(2020)^{10}$ & Turkey & 35 & $24(69)$ & $43.9(12.9)^{*}$ & $0(0)$ & $11(6-17.6)$ \\
\hline Alam et al $(2019)^{20}$ & France & 20 & $10(50)$ & $32(9.6 \mathrm{I})^{*}$ & $20(100)$ & $7.1(2-22.5)$ \\
\hline De Hous et al (2019) ${ }^{19}$ & Belgium & 10 & NS & $50(30-63)$ & $2(20)$ & $9(4-26)$ \\
\hline Stijns et al $(2019)^{17}$ & Netherlands & 20 & $4(20)$ & $45(27-78)^{*}$ & $0(0)$ & $10(7.3)^{* *}$ \\
\hline Marref et al $(2019)^{14}$ & France & 69 & $34(49)$ & $40(33-53)$ & $6(8.7)$ & $6.3(4.2-9.3)$ \\
\hline Terzi et al $(2018)^{22}$ & Turkey & 103 & $82(80)$ & $43(18-78)$ & $0(0)$ & $28(2-50)$ \\
\hline Lauretta et al $(2018)^{11}$ & Italy & 30 & $16(53.3)$ & $52(26-72)$ & $0(0)$ & $11.3(6-24)$ \\
\hline Donmez et al $(2017)^{16}$ & Turkey & 27 & $23(85.2)$ & $35.6(10.32)^{*}$ & NS & $22(17-26)$ \\
\hline Wilhelm et al $(2017)^{15}$ & Germany & 117 & $82(70.1 \%)$ & $46(17-82)$ & $13(11.1)$ & $25.4(6-60)$ \\
\hline \multirow[t]{2}{*}{ Giamundo et al $(2015)^{21}$} & Italy & 45 & $21(47)$ & $46(18-78)$ & $2(4)$ & $30(6-46)$ \\
\hline & & 825 & & & & \\
\hline
\end{tabular}

Notes: *Mean, standard deviation in parenthesis where available; **IQR, inter-quartile range.

Abbreviation: NS, not specified.

assessment in the majority of studies was based on a combination of proctoscopy, endoanal ultrasound and manometry (in selected cases), in the pre-operative period. One study reported routine preoperative magnetic resonance imaging (MRI) in all patients undergoing the procedure, ${ }^{17}$ and Isik et $\mathrm{al}^{13}$ reported that, by performing an MRI prior to surgery and inserting a seton in all cases with an abscess, they could reduce their failure rate from $25 \%$ to $6.6 \%$. $^{13,25}$ The majority of fistulas were complex and these were largely transsphincteric, although the height of the fistula or proportion of sphincter involved was not always reported ${ }^{13,23,26,27}$ (Table 2). Four studies reported treatments in patients with secondary extensions, ${ }^{9,13,19,20}$ and the remainder either excluded patients with this fistula characteristic or did not specify. Eight studies ${ }^{9,12,14,15,18-21}$ reported outcomes in patients with Crohn's-related fistula. One of these studies assessed sole treatment of Crohn's perianal fistulas ${ }^{20}$ in a pilot study of 20 patients; the remaining studies either excluded these patients or included only a few of them (maximum $n=13$ / $117,11 \%{ }^{15}$ ).

\section{Technique Variations}

The majority of the studies performed the $\mathrm{FiLaC}^{\mathrm{TM}}$ procedure using disposable laser fibres and either Ceralas ${ }^{\circledR}$ or, more recently, a Leonardo ${ }^{\circledR}$ DUAL 45 diode laser device form Bio-litec ${ }^{\circledR}$ (Biomedical Technology GmbH, Jena, Germany). Other devices used were the CORONA ${ }^{\mathrm{TM}}$ fistula probe coupled with a neoV $\mathrm{V}^{\mathrm{TM}}$ diode laser system) manufactured by neoLaser ${ }^{\circledR} \quad$ (Endotherapeutics, Australia $^{17}$ and a EUFOTON ${ }^{\circledR}$ diode laser system (Eufoton, Trieste, Italy) coupled with the disposable probe (HF Ring Fiber). ${ }^{11}$ The laser energy used in the studies varied between 10 and 15 Watts and all (except one $^{21}$ ) of the studies used a $1470 \mathrm{~nm}$ wavelength diode laser device. The speed of withdrawal of the fistula probe varied between $1 \mathrm{~mm} / \mathrm{s}$ and $3 \mathrm{~mm} / \mathrm{s}$. 


\begin{tabular}{|c|c|c|c|c|c|c|c|c|c|}
\hline 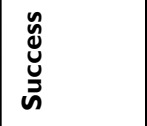 & 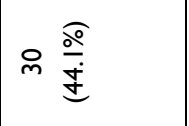 & 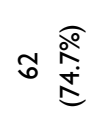 & 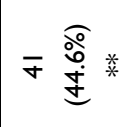 & 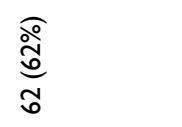 & 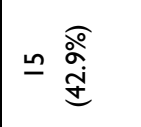 & $\begin{array}{l}\stackrel{\circ}{\stackrel{\circ}{\hat{n}}} \\
= \\
=\end{array}$ & $\stackrel{\circ}{\stackrel{\circ}{ }}$ & 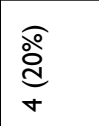 & 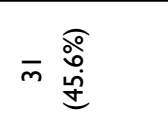 \\
\hline 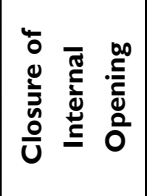 & 站 & 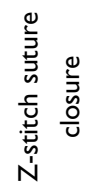 & $\begin{array}{l}\stackrel{0}{\tilde{0}} \\
\text { z }\end{array}$ & $\begin{array}{l}\stackrel{0}{0} \\
\stackrel{0}{z}\end{array}$ & 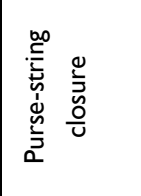 & $\begin{array}{l}\stackrel{0}{0} \\
\stackrel{0}{z}\end{array}$ & 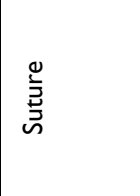 & $\begin{array}{l}\stackrel{0}{0} \\
\stackrel{0}{z}\end{array}$ & $\begin{array}{l}0 \\
\stackrel{0}{0} \\
z\end{array}$ \\
\hline 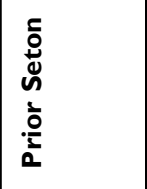 & $\begin{array}{l}\stackrel{\circ}{\circ} \\
\stackrel{0}{\circ} \\
\stackrel{\circ}{0}\end{array}$ & 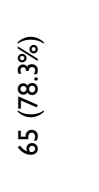 & 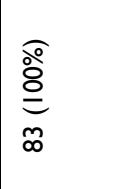 & 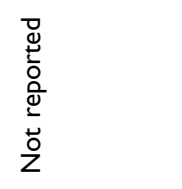 & 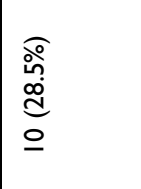 & 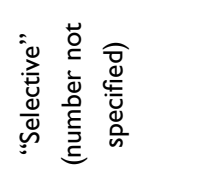 & 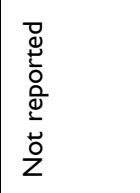 & $\begin{array}{l}\stackrel{0}{\circ} \\
\stackrel{0}{0} \\
\stackrel{0}{0}\end{array}$ & 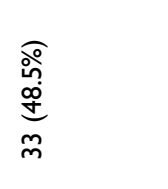 \\
\hline 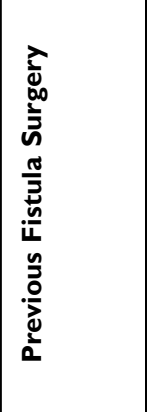 & $\begin{array}{l}\text { స్ల్య } \\
\text { స̃ }\end{array}$ & 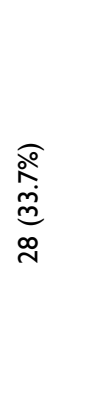 & 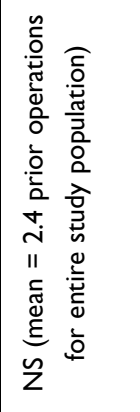 & 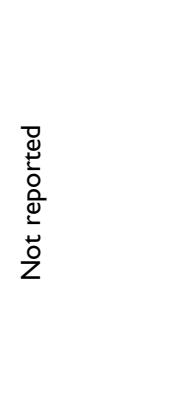 & 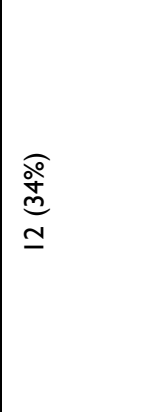 & 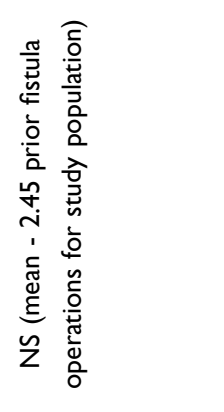 & 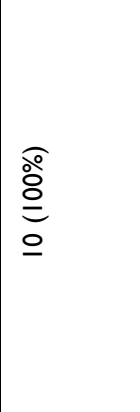 & 1 & 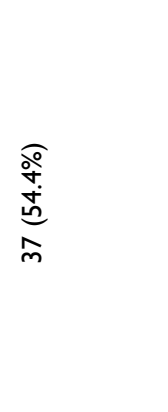 \\
\hline 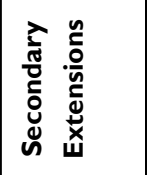 & 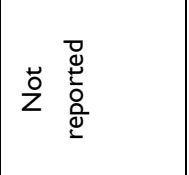 & 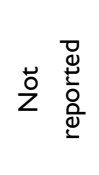 & $\stackrel{\stackrel{\circ}{\stackrel{\rho}{m}}}{\underline{m}}$ & 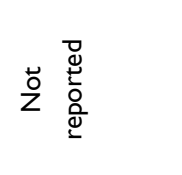 & Z & o్ & 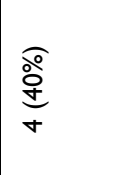 & 0 & $\begin{array}{l}\text { స్ } \\
\text { స̃ } \\
\text { in }\end{array}$ \\
\hline 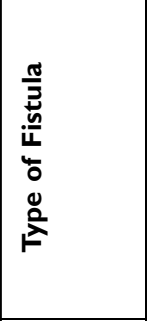 & 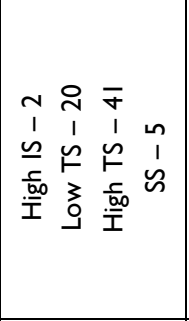 & 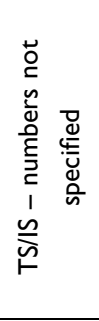 & 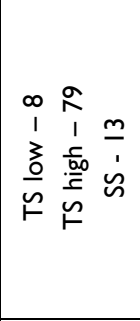 & 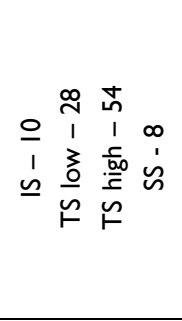 & 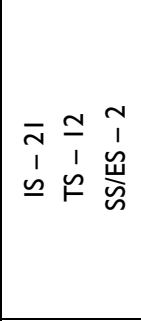 & 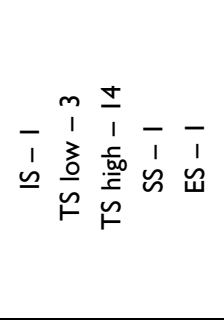 & 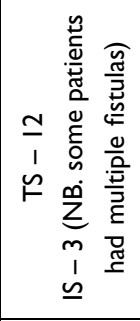 & $\begin{array}{ll}0 & \pm \\
1 & 1 \\
\simeq & \curvearrowleft\end{array}$ & 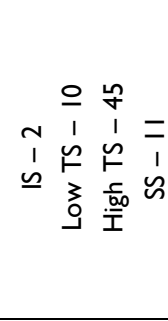 \\
\hline $\begin{array}{l}\frac{n}{0} \\
\stackrel{0}{\xi} \\
\text { ż }\end{array}$ & ๖ & œ & 으 & 으 & $\stackrel{\stackrel{n}{m}}{ }$ & ㅇ & 으 & 이 & $\begin{array}{l}\text { 恙 } \\
\text { 尊 }\end{array}$ \\
\hline 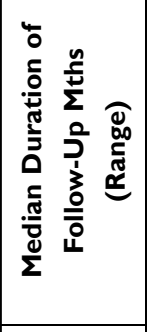 & 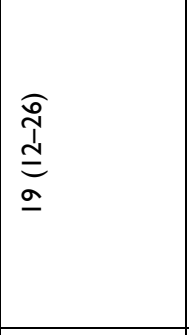 & 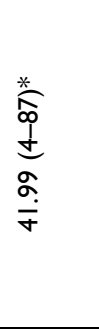 & $\begin{array}{l}\widehat{\hat{I}} \\
\stackrel{1}{0} \\
\stackrel{0}{\underline{m}}\end{array}$ & 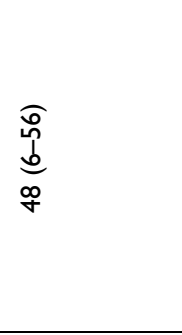 & $\begin{array}{l}\widehat{o} \\
\stackrel{0}{1} \\
e \\
=\end{array}$ & $\begin{array}{l}\frac{\pi}{\mathfrak{I}} \\
\stackrel{1}{d} \\
\frac{\pi}{n}\end{array}$ & $\begin{array}{l}\text { I্ } \\
\text { d } \\
\sigma\end{array}$ & 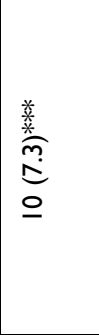 & 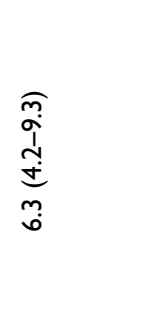 \\
\hline 츨 & 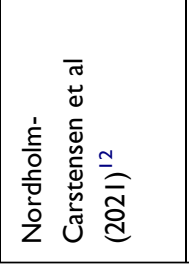 & 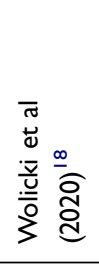 & 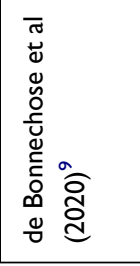 & 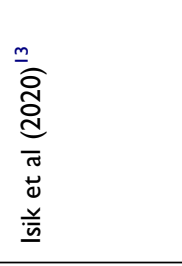 & 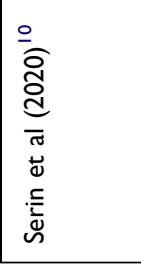 & 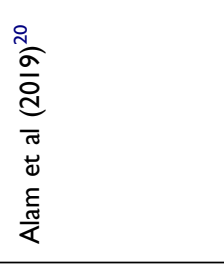 & 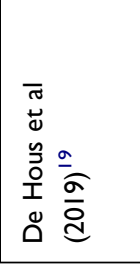 & 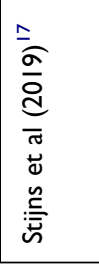 & 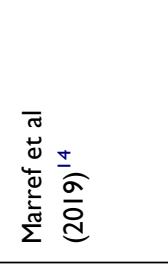 \\
\hline
\end{tabular}




\begin{tabular}{|c|c|c|c|c|}
\hline F & 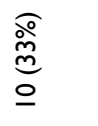 & 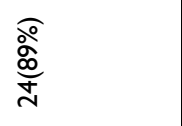 & 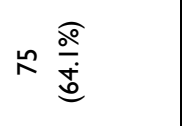 & 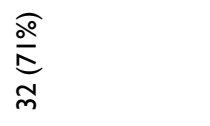 \\
\hline 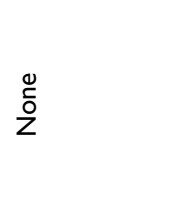 & $\begin{array}{l}\text { 巳̃ } \\
\text { ¿̊ }\end{array}$ & $\begin{array}{l}\text { I0 } \\
\text { Ż }\end{array}$ & 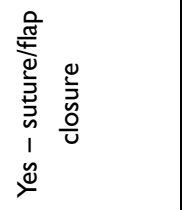 & $\begin{array}{l}\text { ő } \\
\text { ż }\end{array}$ \\
\hline 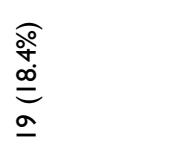 & 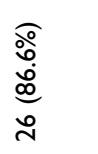 & 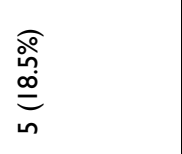 & 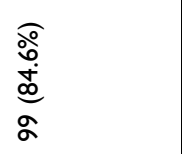 & 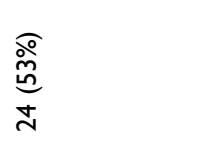 \\
\hline 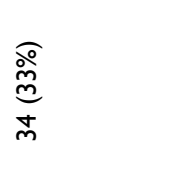 & 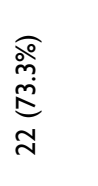 & 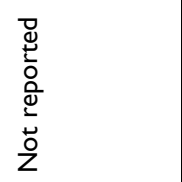 & 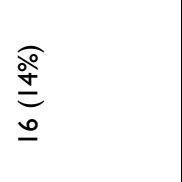 & 总 \\
\hline 荾 & 0 & $z$ & 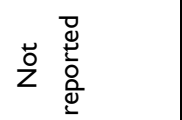 & 0 \\
\hline 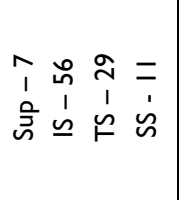 & $\begin{array}{l}o \\
\dot{m} \\
\dot{\varphi}\end{array}$ & 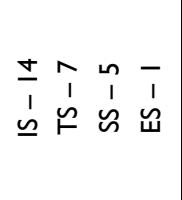 & $\begin{array}{llll}\infty & \& & m & 0 \\
1 & 1 & 1 & 1 \\
\underline{\omega} & \sim & \sim & \tilde{\omega}\end{array}$ & 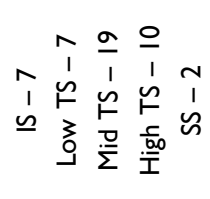 \\
\hline$\underline{\cong}$ & ᄋ & $\hat{\wedge}$ & $\triangleq$ & ६ \\
\hline 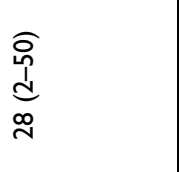 & $\begin{array}{l}\underset{\mathbb{N}}{\mathfrak{d}} \\
\underline{m} \\
\stackrel{m}{=}\end{array}$ & 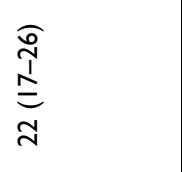 & 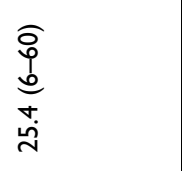 & $\begin{array}{l}\widehat{o} \\
1 \\
0 \\
0 \\
0\end{array}$ \\
\hline 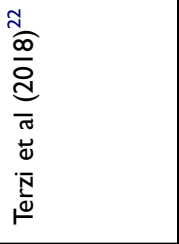 & 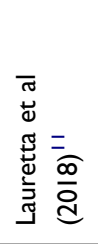 & 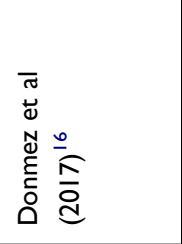 & 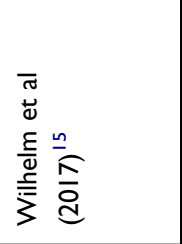 & 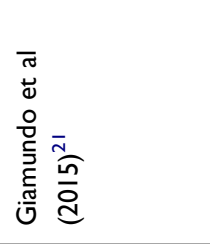 \\
\hline
\end{tabular}


The internal opening was not closed in the majority of studies..$^{9,11,13,14,16,17,20-22}$ Four studies ${ }^{10,12,18,19}$ closed the internal opening with sutures and one study reported closure with a combination advancement flap and sutures. ${ }^{15}$

\section{Outcomes}

Nordholm-Carstensen et $\mathrm{al}^{12}$ followed up all patients with a clinical examination including MRI or endo-anal ultrasound (EAUS) one year after the procedure. A few studies also used EAUS, ${ }^{11,15}$ with some restricting imaging to assessing cases of failure or recurrence. ${ }^{11,21}$ In most studies, however, healing was assessed clinically, without radiological confirmation.

Isik et al recently reported the longest median follow-up of 48 months (range 6-56 months) in a cohort of 100 patients treated, whilst Wolicki et al reported a mean followup of 42 months ( \pm SD 21.34; range 4.8-87.6 months).

Success rates, ie primary healing (mostly assessed clinically), varied from $20 \%(4 / 20)^{17}$ at a median followup of 10 months to $89 \%(24 / 27)^{16}$ reported by Donmez et al at a median follow-up of 22 months (Table 2). Seven of the studies reported success rates (fistula healing) in fewer than $50 \%$ of patients, ${ }^{9-12,14,17,22}$ whereas the remaining seven studies reported healing rates in more than $55 \%$ of the patients treated (Table 2). The four largest studies (including more than 100 patients) reported primary healing rates of $44 \%$ (41/92 patients followed up at a median of 13.6 months) $;{ }^{9} 40 \%$ (41/103 patients followed up at a median of 28 months); ${ }^{22} 62 \%(62 / 100$ patients followed up at a median of 48 months) and $64 \%$ (75/117 patients followed up at a median of 25.4 months).

Analysis of those studies reporting healing rates for FiLaC in "high" transsphincteric fistulas revealed a success rate of $54 \%(118 / 219)$ in cryptoglandular fistulas and one study reported healing in 71\% (10/14) in Crohn's perianal fistula.

\section{Crohn's Disease}

- Alam et $\mathrm{al}^{20}$ reported a success rate of $55 \%$, with fistula healing noted in 11/20 patients with Crohn's perianal fistulas at a median follow-up period of 7.1 months. Overall, a very small minority of all patients in the studies considered had Crohn's perianal fistulas $(66 / 825 ; 8 \%)$. Of the 66 patients, $39(59.1 \%)$ were reported to have undergone fistula healing.

\section{Complications/Adverse Effects}

Few complications were reported following laser treatment of perianal fistulas in the studies assessed. There were no reports of any deterioration in faecal continence. Eight studies reported the absence of any morbidity following the procedure. ${ }^{9,10,12-14,16,19,20,22}$ Giamundo et $\mathrm{al}^{21}$ reported eight $(8 / 43,18 \%)$ patients with temporary pain and anismus postoperatively and three $(3 / 43,7 \%)$ patients with moderate bleeding after FiLaC treatment. All of these resolved without intervention. Similarly, Lauretta et $\mathrm{al}^{11}$ reported four patients with minor postoperative complications (two with fever, one with severe pain and one with moderate bleeding) that resolved spontaneously. Stijns et $\mathrm{al}^{17}$ reported four patients $(20 \%, 4 / 20)$ who developed perianal abscess (one within 30 days, one at six weeks postoperatively and the remaining two more than six months after the procedure). Stijns et al also reported a postoperative deterioration in patient reported anorectal function in seven patients, when measured using the Faecal Incontinence Severity Index (FISI). Wolicki et a ${ }^{18}$ reported minor complications: 11 patients $(11 / 83,13 \%)$ developed pain and $7(7 / 83,8 \%)$ developed bleeding postoperatively.

\section{Discussion}

Lay-open remains the optimum means of curing a fistula. However, when the fistula involves the muscle, the benefit of cure must be balanced by the risk to the patient's continence. There remains no optimum technique to negate this risk of incontinence, thus stimulating an array of surgical techniques to address this issue. However, none of these procedures guarantees a good result, and for this reason new techniques have been introduced. FiLaC has now been reported in over 600 patients and its feasibility and safety have been demonstrated in these studies. The efficacy from the literature is somewhat variable, ranging from $20 \%$ to $89 \%$ rates of fistula healing. There are no reports of faecal incontinence and the ability to repeat the procedure in the face of minimal morbidity makes it an attractive prospect in the context of sphincter-sparing fistula surgery.

Some of the success rates reported in the FiLaC studies $^{16,18,19,26}$ compare satisfactorily with those of other sphincter-sparing techniques. ${ }^{5,28-30}$ Recent systematic reviews reveal pooled healing rates of approximately $70 \%$ for the LIFT procedure in almost 500 patients with perianal fistula. ${ }^{28,30}$ In advancement flaps, a pooled success rate of approximately $75 \%$ has been reported in analysis of close to 800 patients. $^{28}$ It remains to be seen whether these initially high success rates reported by some of the FiLaC procedure results will stand the test of time in 
the upcoming years. There have already been some studies suggesting much more moderate/doubtful benefits. ${ }^{11,31}$

There were some notable differences between the reported studies of FiLaC, particularly the addition of the closure of the internal opening (by suture or advancement flap) that was employed in some cohorts. ${ }^{10,12,15,18,19}$ This combination has been questioned in prior literature, ${ }^{17,31}$ with the suggestion that the combination of two treatment strategies limits the ability to critique the impact of FiLaC alone on the outcome of the treatment. Furthermore, some studies reporting on FiLaC in the absence of treatment of the internal opening have reported better outcomes, ${ }^{13,16,26}$ thus questioning the value of the additional closure. The target patient cohort that would derive benefit from this procedure is difficult to discern in view of the heterogeneity in the studies and indeed the outcomes. The majority of patients had transsphincteric fistulas, but their height and complexity were not always clearly reported. Indeed this variability in type, length and size of the fistulas has been proposed as potentially contributing to the different outcomes. ${ }^{32}$ Further work is being undertaken to highlight the heterogeneity of outcome reporting and develop a core outcome set in this context. ${ }^{33,34}$

Lauretta et $\mathrm{al}^{11}$ reported in their case series $(\mathrm{n}=30)$ that the only significant prognostic factor identified by statistical analysis was fistula length. The mean fistula length in the group of patients who were cured by fistula laser closure was $28.5 \mathrm{~mm}(10-41 \mathrm{~mm}$; SD 12.4), while it was $46 \mathrm{~mm}(25-67 \mathrm{~mm}$; SD 20.6) in those not cured. Fistula tracts shorter than $30 \mathrm{~mm}$ were associated with a primary healing rate of $58.3 \%$ while tracts longer than $30 \mathrm{~mm}$ were cured in only $16.6 \%$ of cases $(p<0.02)$. However, this is not consistent with other studies that have not corroborated this finding ${ }^{9,12,14,32}$ and longer tracts have been suggested to improve the shrinkage effect elicited by laser energy. ${ }^{32}$ There have been suggestions that prior seton drainage may be beneficial ${ }^{21,32}$ by aiding fistula "maturation" towards an optimum width, but again this was not unanimously employed and has not been consistently shown to be associated with better outcomes. There is no data about the optimal time for the seton to be in place or whether an advantage is conferred at all.

\section{Strengths and Limitations of FiLaC and Studies}

The FiLaC technique is minimally invasive and has been reported to be performed in outpatient settings in some series. ${ }^{11,21,22}$ There have been promising reports of success (in terms of primary fistula healing) and it can be performed multiple times with seemingly minimal impact on continence. It does, however, come with a significant cost implication when compared with other techniques. Anatomy of the fistula tract may present problems, and presence of secondary extensions may not always be accessible by the laser fistula probe. Also, very few studies adopt routine pre- and postoperative MRI to confirm tract anatomy and subsequent healing. This may affect success of the technique as hidden tracts and unidentified extensions are a common cause of failure for all fistula surgery. Furthermore, clinical healing may not always constitute radiological healing and this has been described often in the context of Crohn's perianal fistulas, whereby radiological healing may lag behind for up to a year. ${ }^{35}$ In more complex fistula anatomy, it may be necessary for FiLaC to be used as a complement to other techniques, and indeed its combination with video-assisted anal fistula surgery (VAAFT) has recently been described. ${ }^{27}$ Further limitations of studies reporting on FiLaC are their retrospective nature, with single-centre data including heterogeneous study populations, which limits the external validity and reproducibility of the results.

\section{Conclusion}

The success and continence risks associated with laying open simple fistula mean that newer techniques need to be explored and assessed for superiority, both in terms of patient outcomes and cost benefit. The laser procedure is demonstrably feasible and appears a relatively easy to learn technique and has been demonstrated to be safe with no reports of faecal incontinence. The target patient population that would derive most benefit appears to be those with more complex fistulas or recurrent fistulas where a lay-open cannot be considered due to sphincter involvement and risks of incontinence. The impact of prior seton drainage or specific closure of the internal opening may play a role in the healing rate, although this is not proven. Further studies are still warranted to discern the role of laser therapy in the armamentarium of fistula surgery and comparisons with surgeons' preferences (similar to the FIAT study ${ }^{36}$ ) will aid in this pursuit.

\section{Author Contributions}

All authors contributed to data analysis, drafting or revising the article, gave final approval of the version to be 
published, agreed to the submitted journal, and agree to be accountable for all aspects of the work.

\section{Funding}

There is no funding to report.

\section{Disclosure}

The authors report no conflicts of interest in this work.

\section{References}

1. Mizrahi N, Wexner SD, Zmora O, et al. Endorectal advancement flap: are there predictors of failure? Dis Colon Rectum. 2002;45 (12):1616-1621. doi:10.1097/01.DCR.0000037654.01119.CD

2. Rojanasakul A. LIFT procedure: a simplified technique for fistula-inano. Tech Coloproctol. 2009;13(3):237-240. doi:10.1007/s10151009-0522-2

3. Meinero P, Mori L. Video-assisted anal fistula treatment (VAAFT): a novel sphincter-saving procedure for treating complex anal fistulas. Tech Coloproctol. 2011;15(4):417-422. doi:10.1007/s10151-0110769-2

4. Wilhelm A. A new technique for sphincter-preserving anal fistula repair using a novel radial emitting laser probe. Tech Coloproctol. 2011;15(4):445-449. doi:10.1007/s10151-011-0726-0

5. Adegbola SO, Sahnan K, Pellino G, et al. Short-term efficacy and safety of three novel sphincter-sparing techniques for anal fistulae: a systematic review. Tech Coloproctol. 2017;21(10):775-782. doi:10.1007/s10151-017-1699-4

6. Ellison GW, Bellah JR, Stubbs WP, Van Gilder J. Treatment of perianal fistulas with ND:YAG laser-results in twenty cases. Vet Surg. $\quad 1995 ; 24(2): 140-147 . \quad$ doi:10.1111/j.1532-950x.1995. tb01308.x

7. Bodzin JH. Laser ablation of complex perianal fistulas preserves continence and is a rectum-sparing alternative in Crohn's disease patients. Am Surg. 1998;64(7):627-632.

8. Bass LS, Treat MR, Dzakonski C, Trokel SL. Sutureless microvascular anastomosis using the THC:YAG laser: a preliminary report. Microsurgery. 1989;10(3):189-193. doi:10.1002/ micr.1920100309

9. de Bonnechose G, Lefevre JH, Aubert M, et al. Laser ablation of fistula tract (LAFT) and complex fistula-in-ano: "the ideal indication" is becoming clearer.... Tech Coloproctol. 2020;24(7):695-701. doi:10.1007/s10151-020-02203-y

10. Serin KR, Hacim NA, Karabay O, Terzi MC. Retrospective analysis of primary suturing of the internal orifice of perianal fistula during FiLaC procedure. Surg Laparosc Endosc Percutan Tech. 2020;30 (3):266-269. doi:10.1097/SLE.0000000000000774

11. Lauretta A, Falco N, Stocco E, Bellomo R, Infantino A. Anal fistula laser closure: the length of fistula is the achilles' heel. Tech Coloproctol. 2018;22(12):933-939. doi:10.1007/s10151-018-1885-Z

12. Nordholm-Carstensen A, Perregaard H, Hagen KB, Krarup P-M. Fistula Laser Closure (FiLaC ${ }^{\mathrm{TM}}$ ) for fistula-in-ano-yet another technique with $50 \%$ healing rates? Int J Colorectal Dis. 2021. doi:10.1007/s00384-021-03932-8

13. Isik O, Gulcu B, Ozturk E. Long-term outcomes of laser ablation of fistula tract for fistula-in-ano: a considerable option in sphincter preservation. Dis Colon Rectum. 2020;6:831-836. doi:10.1097/ DCR.0000000000001628

14. Marref I, Spindler L, Aubert M, et al. The optimal indication for $\mathrm{FiLaC}^{\circledR}$ is high trans-sphincteric fistula-in-ano: a prospective cohort of 69 consecutive patients. Tech Coloproctol. 2019;23(9):893-897. doi:10.1007/s10151-019-02077-9
15. Wilhelm A, Fiebig A, Krawczak M. Five years of experience with the $\mathrm{FiLaC}^{\mathrm{TM}}$ laser for fistula-in-ano management: long-term follow-up from a single institution. Tech Coloproctol. 2017;21(4):269-276. doi:10.1007/s10151-017-1599-7

16. Dönmez T, Hatipoğlu E. Closure of fistula tract with filac ${ }^{\mathrm{TM}}$ laser as a sphincter preserving method in anal fistula treatment. Turk $J$ Color Dis. 2018. doi: $10.4274 / \mathrm{tjcd} .06025$

17. Stijns J, van Loon YT, Clermonts SHEM, Göttgens KW, Wasowicz DK, Zimmerman DDE. Implementation of laser ablation of fistula tract (LAFT) for perianal fistulas: do the results warrant continued application of this technique? Tech Coloproctol. 2019;23 (12):1127-1132. doi:10.1007/s10151-019-02112-9

18. Wolicki A, Jäger P, Deska T, Senkal M. Sphincter-saving therapy for fistula-in-ano: long-term follow-up after $\mathrm{FiLaC}^{\circledR}$. Tech Coloproctol. 2020;25(2):177-184. doi:10.1007/s10151-020-02332-4

19. De Hous N, de Gheldere C, Van den Broeck S, Komen N. FiLaC ${ }^{\mathrm{TM}}$ as a last, sphincter-preserving resort for complex perianal fistula. Tech Coloproctol. 2019;23(9):937-938. doi:10.1007/s10151-01902070-2

20. Alam A, Lin F, Fathallah N, et al. FiLaC ${ }^{\circledR}$ and Crohn's disease perianal fistulas: a pilot study of 20 consecutive patients. Tech Coloproctol. 2020;24(1):75-78. doi:10.1007/s10151-019-02134-3

21. Giamundo P, Esercizio L, Geraci M, Tibaldi L, Valente M. Fistulatract Laser Closure (FiLaCTM): long-term results and new operative strategies. Tech Coloproctol. 2015;19(8):449-453. doi:10.1007/ s10151-015-1282-9

22. Terzi MC, Agalar C, Habip S, Canda AE, Arslan NC, Obuz F. Closing perianal fistulas using a laser: long-term results in 103 patients. Dis Colon Rectum. 2018;61(5):599-603. doi:10.1097/ DCR.0000000000001038

23. Frountzas M, Stergios K, Nikolaou C, et al. Could FiLaC ${ }^{\mathrm{TM}}$ be effective in the treatment of anal fistulas? A systematic review of observational studies and proportional meta-analysis. Color Dis. 2020;22(12):1874-1884. doi:10.1111/codi.15148

24. Elfeki H, Shalaby M, Emile SH, Sakr A, Mikael M, Lundby L. A systematic review and meta-analysis of the safety and efficacy of fistula laser closure. Tech Coloproctol. 2020;24(4):265-274. doi:10.1007/s10151-020-02165-1

25. Öztürk E, Gülcü B. Laser ablation of fistula tract. Dis Colon Rectum. 2014;57(3):360-364. doi:10.1097/DCR.0000000000000067

26. Giamundo P, Geraci M, Tibaldi L, Valente M. Closure of fistula-inano with laser-FiLaC ${ }^{\mathrm{TM}}$ : an effective novel sphincter-saving procedure for complex disease. Colorectal Dis. 2014;16(2):110-115. doi:10.1111/codi. 12440

27. Yao YB, Xiao CF, Wang QT, et al. VAAFT plus FiLaC ${ }^{\mathrm{TM}}$ : a combined procedure for complex anal fistula. Tech Coloproctol. 2021;0123456789:1-3. doi:10.1007/s10151-021-02411-0

28. Stellingwerf ME, van Praag EM, Tozer PJ, Bemelman WA, Buskens CJ. Systematic review and meta-analysis of endorectal advancement flap and ligation of the intersphincteric fistula tract for cryptoglandular and crohn's high perianal fistulas. BJS Open. 2019;3 (3):231-241. doi:10.1002/bjs5.50129

29. Alasari S, Kim NK. Overview of anal fistula and systematic review of ligation of the intersphincteric fistula tract (LIFT). Tech Coloproctol. 2014;18(1):13-22. doi:10.1007/s10151-013-1050-7

30. Yassin NA, Hammond TM, Lunniss PJ, Phillips RKS. Ligation of the intersphincteric fistula tract in the management of anal fistula. A systematic review. Color Dis. 2013;15(5):527-535. doi:10.1111/ codi. 12224

31. Stijns J, Wasowicz DK, Zimmerman DDE. Does laser fistuloplasty $\left(\mathrm{FiLaC}^{\mathrm{TM}}\right)$ offer any benefit over surgical closure of the internal orifice? Tech Coloproctol. 2017;21(6):489-490. doi:10.1007/ s10151-017-1626-8

32. Giamundo P. Laser treatment for anal fistulas: what are the pitfalls? Tech Coloproctol. 2020;24(7):663-665. doi:10.1007/s10151-02002225-6 
33. Machielsen AJHM, Iqbal N, Kimman ML, et al. Heterogeneity in Outcome Selection, Definition and Measurement in Studies Assessing the Treatment of Cryptoglandular Anal Fistula: Findings from a Systematic Review. Vol. 25. Springer International Publishing; 2021. doi:10.1007/s10151-021-02452-5

34. Machielsen AJHM, Iqbal N, Kimman ML, et al. The development of a cryptoglandular Anal Fistula Core Outcome Set (AFCOS): an international Delphi study protocol. United Eur Gastroenterol J. 2020;8(2):220-226. doi:10.1177/2050640620907570
35. Tozer P, Ng SC, Siddiqui MR, et al. Long-term MRI-guided combined anti-TNF- $\alpha$ and thiopurine therapy for Crohn's perianal fistulas. Inflamm Bowel Dis. 2012;18(10):1825-1834. doi:10.1002/ibd.21940

36. Jayne DG, Scholefield J, Tolan D, et al. Anal fistula plug versus surgeon's preference for surgery for trans-sphincteric anal fistula: the FIAT RCT. Health Technol Assess. 2019;23(21):1-76. doi:10.3310/ hta23210

\section{Publish your work in this journal}

Clinical and Experimental Gastroenterology is an international, peerreviewed, open access, online journal publishing original research, reports, editorials, reviews and commentaries on all aspects of gastroenterology in the clinic and laboratory. This journal is indexed on American Chemical Society's Chemical Abstracts Service (CAS)
The manuscript management system is completely online and includes a very quick and fair peer-review system, which is all easy to use. Visit http://www.dovepress.com/testimonials.php to read real quotes from published authors.

Submit your manuscript here: https://www.dovepress.com/clinical-and-experimental-gastroenterology-journal 